\title{
In silico design of colchicine-based bioisosteric inhibitors of tubulin for the treatment of rheumatoid arthritis
}

\author{
LI WANG ${ }^{1}$, HAO-JIE YANG ${ }^{2}$, RAN TU $^{3}$, MIN SHEN $^{4}$ and DAN LIU ${ }^{1,5}$ \\ ${ }^{1}$ Department of Rheumatology and Immunology, Rheumatology Institute, The Fifth Hospital of Xi'an, Xi'an, \\ Shaanxi 710082; ${ }^{2}$ Department of Prevention and Health Care, Xi'an Jiaotong University Hospital, Xi'an, Shaanxi 710049; \\ ${ }^{3}$ College of Life Sciences, Shaanxi Normal University College of Life Sciences, Xi'an, Shaanxi 710119; \\ ${ }^{4}$ Department of Respiratory Medicine, Northwest Petroleum Pipeline Hospital, Xi'an, Shaanxi 710082; \\ ${ }^{5}$ Core Research Laboratory, The Second Affiliated Hospital of Xi'an Jiaotong University, Xi'an, \\ Shaanxi 710004, P.R. China
}

Received May 28,2016; Accepted April 6, 2017

DOI: $10.3892 / \mathrm{mmr} .2017 .7202$

\begin{abstract}
The super-saturation of serum with monosodium urate due to hyperuricemia is the core metabolic disorder of rheumatoid arthritis. When the serum urate concentration is $\geq 7 \mathrm{mg} / \mathrm{dl}$, this results in the crystallization of monosodium urate in serum at body temperature $\left(37^{\circ} \mathrm{C} / 98.6^{\circ} \mathrm{F}\right)$. Colchicine (COL) is considered to be a first-line medication for acute arthritis when NSAIDs are contraindicated. COL causes severe side effects, including diarrhea, nausea, cramping, abdominal pain and vomiting, in humans. Experimental studies have additionally demonstrated the presence of mutagenic and reproductive effects in humans. In the present study, molecular docking simulation techniqueswere used to design COL-derived bioisosteric inhibitors, with the aim of designing an alternative treatment that exhibitedpotent anti-arthritic activity and was free from the side effects associated with COL.
\end{abstract}

\section{Introduction}

Rheumatoid arthritis is a painful disease condition characterized by an increased serum urate concentration. Recurrent acute arthritic attacks are associated with the presence of monosodium urate crystals in the leukocytes of synovial fluid. These monosodium urate crystals are termed tophi, and are deposited in the tissues around joints, although they may additionally cause interstitial renal disease and uric acid nephrolithiasis (1). Causes of acute arthritic attacks include surgery, alterationsin meteorological conditions, emotional

Correspondence to: Mr Dan Liu, Department of Rheumatology and Immunology, Rheumatology Institute, The Fifth Hospital of Xi'an, 112 West Center Street, Xi'an, Shaanxi 710082, P.R. China E-mail: liudan600@hotmail.com

Key words: rheumatoid arthritis, colchicine, molecular docking, ligand, bioisosteres upset, treatment with diuretic and antibiotic drugs, and parenteral administration of penicillin and vitamin B (1).

The initial attack of acute arthritis is commonly observed in the middle decades of life, although it may present in early or later life stages. Acute arthritis may manifest spontaneously, or following the impact of one of numerous precipitating agents. The super-saturation of serum with monosodium urate due to hyperuricemia is the core metabolic disorder of arthritis (2). When the serum urate concentration of serum exceeds $\sim 7 \mathrm{mg} / \mathrm{dl}$, this results in the crystallization of monosodium urate in serum at body temperature $\left(37^{\circ} \mathrm{C} / 98.6^{\circ} \mathrm{F}\right)(2)$. The risk of arthritis in a patient is indicated by the degree of hyperuricemia; statistically, hyperuricemia is defined as a serum urate concentration $>2$ standard deviations above the population means for age and sex-matched healthy populations, which is typically $7 \mathrm{mg} / \mathrm{dl}$ for men and $6 \mathrm{mg} / \mathrm{dl}$ for women (2). However, the presence of hyperuricemia isfrequently an asymptomatic condition which leads to the development of arthritis (2).

The uric acid produced by the metabolism of amino acids originates from three primary sources: Dietary purine; de-novo synthesis of purine bases; and conversion of purine nucleotides from tissue nucleic acid. Purines derived from all of the three sources commonly enter the same metabolic pathway, resulting in the production of nucleic acid oruric acid (2). Under normal conditions, when the production rate of uric acid synthesized by the metabolic pathway exceeds its excretion rate, it may begin to accumulate excessively in the surrounding joints and tissues (2).

Purine metabolism is regulated by a number of enzymatic systems. The over-production of uric acid results from abnormal alterations in these regulatory systems. Uric acid may be over-produced as a consequence of the increased breakdown of nucleic acids and excessive cell turnover rates, as a result of myelo-proliferative and lympho-proliferative disorders, polycythemiavera, psoriasis, and certain types of anemia $(3,4)$. Cytotoxic medications used to treat these disorders may result in the over-production of uric acid followed by thelysis and breakdown of cellular components $(3,4)$. Over-activity of phosphoribosyl pyrophosphate (PRPP) synthetase enzyme results in an increased concentration of PRPP, which is a determinant 
of purine synthesis and is responsible for the production of uric acid $(3,4)$. Under-activity of the hypoxanthine-guanine phosphoribosyltransferase (HGPRT) enzyme is associated with over-production of uric acid. HGPRT converts guanine and hypoxanthine to guanylic acid and inosinic acid, respectively $(3,4)$. PRPP acts as the co-substrate in these two conversions, which are important reactions for the synthesis of nucleic acids. Underactivity of HGPRT leads toincreased metabolism of guanine and hypoxanthine to uric acid, and PRPP interacts with glutamine in the initial stage of the purine pathway $(3,4)$. A complete absence of HGPRT causes choreoathetosis, spasticity, mental retardation, and excessive production of uric acid, characteristics of childhood Lesch-Nyhan syndrome. A partial deficiency of HGRPT may cause marked hyperuricemia in healthy individuals $(3,4)$.

The majority of patients (80-90\%) with arthritis exhibit relative decreases inthe renal excretion of uric acid, for an unknown reason (termed primary idiopathic hyperuricemia). Typically, the accumulation of uric acid does not occur until the production is balanced with the elimination $(3,4)$. There are two ways of excreting uric acid: Approximately two-thirds of the uric acid produced daily is excreted via urine, while the remaining uric acid is degraded by the enzymatic activity of colonic bacteria and eliminated through the gastrointestinal tract. A decrease in uric acid excretion through urine, with respect to the rate of uric acid production, may lead to hyperuricemia and an increased miscible pool of sodium urate. The majority of the urate in plasma is filtered freely across the glomerulus $(3,4)$. The concentration of uric acid in the urine is determined by multiple renal tubular transport processes, in addition to the filtered load. Previous studies have led to the hypothesized four component model, including glomerular filtration, tubular reabsorption, tubular secretion and post-secretory reabsorption. A total of $\sim 90 \%$ of filtered uric acid is reabsorbed in the proximal tubule, likely by active and passive transport mechanisms. An association exists between proximal tubular sodium reabsorption and uric acid reabsorption; therefore, conditions that enhance sodium reabsorption (e.g. dehydration) lead to increased uric acid reabsorption $(3,4)$. The exact site of tubular secretion of uric acid has not been determined; this may additionally involve an active transport process. Post-secretory reabsorption occurs at a site distal to the secretory site $(3,4)$.

Drug design is an iterative and complex process, beginning with the identification of the biological profile of interest, and ending with the optimization of the chemical synthesis of a new chemical entity with that activity profile. Traditional approaches used to discover a new drug depend on step-wise synthesis and biological screening for optimization of pharmacological activity profiles (4-8). Previous studies have used various computer models of novel chemical entities in order to predict their activity profile, geometry and reactivity (4-8).

The development of modern computer technology and methodologies to calculate molecular properties have made it increasingly possible to use computer techniques to aid the drug discovery process $(5,6)$. The use of computer techniques in this context is frequently termed computer-aided drug design. If the 3D structure of the target enzyme or receptor is available from X-ray crystallography, preferentially with a co-crystallized ligand demonstrating the binding site and mode of the ligand, it is feasible to study the bio-macromolecule-ligand complex in a direct way using interactive computer graphic techniques and computational chemistry $(5,6)$. Therefore, knowledge of the interactions between the ligand and the enzyme/receptor may be obtained. Novel candidate ligands may be 'docked' into the binding site in order to investigate whether the structure is able to interact with the receptor in an optimal way. This procedure is known as structure-based ligand design (5-8).

Molecular docking is the process of binding a ligand to the binding site of the receptor by mimicking the natural course of the interaction of the ligand and its receptor via the lowest energy pathway. The docking of rigid ligands with rigid receptors, and flexible ligands with rigid receptors, is simple to perform, although the methods used for the docking of conformationally flexible ligands and receptors are complex and problematic (5-8).

Colchicine (COL) is a medicine used to treat rheumatoid arthritis, as it prevents white blood cells from travelling into the affected areas, and therefore helps to reduce pain and tenderness (Table I). COL is indicated for acute arthritic attacks. It is considered to be a first-line medication for acute arthritis when NSAIDs are contraindicated. It is hypothesized that colchicine interferes with the cell division process through disruption of the mitotic spindle. The potential mechanisms of action of COL have been demonstrated using polarized light microscopy, and through isolation of the remnants of the mitotic apparatus of colchicine-treated seaurchin eggs. COL-mediated inhibition of the mitotic spindlemay occur through a direct or indirect effect of the compound (2-5). To date, COL has primarily been applied to effectively treat gouty arthritis however, although rheumatoid and gouty arthritis are two different diseases, they induce similar symptoms, and so novel derivative molecules of COLs may also be effective against rheumatoid arthritis (2-5).

COL is associated with side effects, including diarrhea, nausea, cramping, abdominal pain and vomiting. Experimental studies have demonstrated the presence of mutagenic and reproductive effects in humans following treatment with COL. Therefore, further studies are required to develop an alternative drug for the treatment of rheumatoid arthritis (1-5).

\section{Materials and methods}

Molecular docking simulations. All calculations were performed using Autodock (version 4.2) as a docking tool (8-12). Visualization and other procedures in the docking studies were performed using PyMOL (pymol.org), Chimera (cgl.ucsf.edu/chimera), Discovery StudioVisualizer v3.5 (DassaultSystèmes BIOVIA, San Diego, CA, USA) and Molecular Modelling ProPlus (version 7.0; Norgwyn Montgomery Software, Inc., North Wales, PA, USA).

\section{Docking of tubulin receptor with colchicine}

Crystal structure. The crystal structure of the protein, consisting of the receptor associated with a bound ligand, was downloaded from the Research Collaboratory of Structural Bioinformatics Protein Data Bank (13). All of the primary information regarding the receptor and structure (identification no. 3E22.pdb) registered in the Protein Data Bank was used. 
Table I. Results of docking of colchicine and its derivatives.

\begin{tabular}{|c|c|c|c|c|}
\hline No. & Compound name & Structure & $\mathrm{Ki}, \mu \mathrm{M}$ & Binding energy, $\mathrm{kcal} / \mathrm{mol}$ \\
\hline 1 & Colchicine & & 5.95 & -7.13 \\
\hline 2 & COL_1 & & 10.94 & -6.77 \\
\hline 3 & COL_2 & & 9.18 & -6.87 \\
\hline 4 & COL_3 & & 13.18 & -6.66 \\
\hline 5 & COL_4 & & 69.29 & -5.67 \\
\hline 6 & COL_5 & & 20.19 & -6.40 \\
\hline
\end{tabular}

$\mathrm{Ki}$, inhibition constant; COL, colchicine.

The bound ligand COL was identified within the receptor. All of the chains of the downloaded protein with the bound ligand COL are presented in Fig. 1.

Processing of protein. The downloaded receptor protein exhibited five chains, termed chains A-E. From these five chains, chain B was selected for experimentation and the remaining chains were removed. Chain B of the downloaded protein contained the bound ligand COL, which was separated using Chimera software (14). The separated chain B of the tubulin receptor protein is presented in Fig. $2 \mathrm{~A}$, while COL is presented in Fig. 2B. 


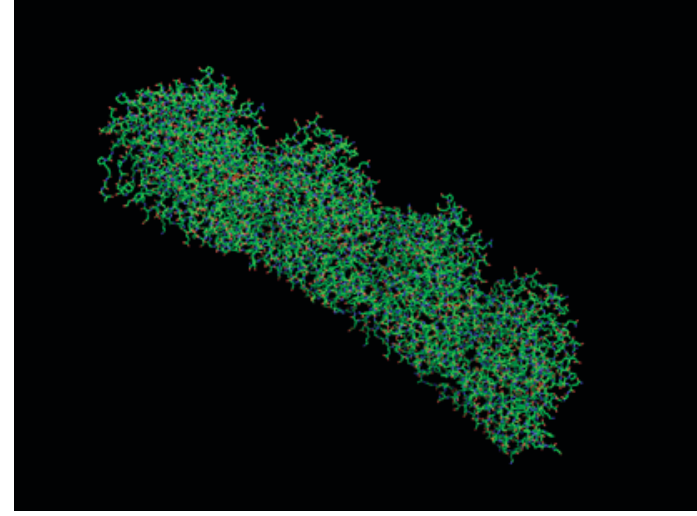

Figure 1. Crystal structure of the human tubulin receptor of the human, exhibiting five chains and bound with the ligand colchicine. The structure was obtained from the Protein Data Bank (identification no. 3E22.pdb) at a resolution of $3.8 \AA$.

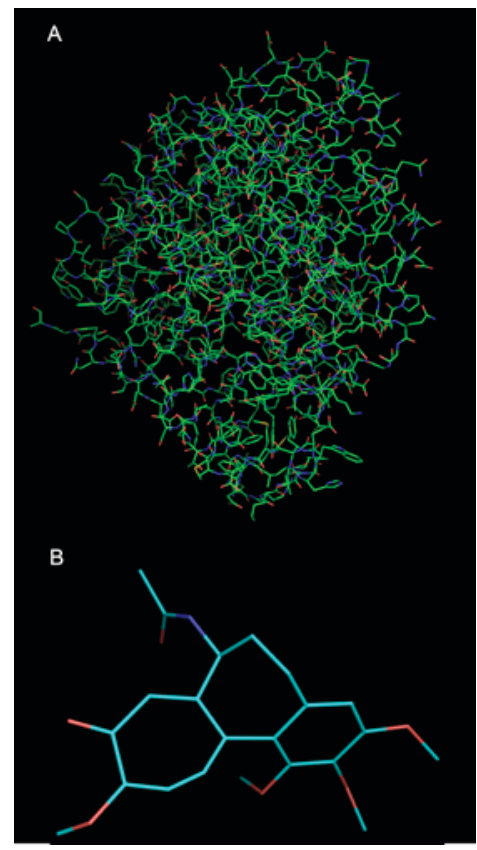

Figure 2. Separation of colchicine ligand from tubulin protein. (A) Chain B of tubulin protein was selected for molecular docking simulation studies and wasseparated from the other chains. (B) The bound ligand colchicine was separated from chain B of tubulin.

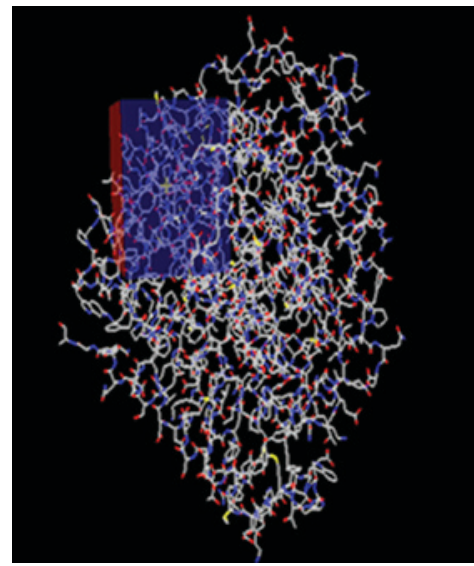

Figure 3. Three-dimensional grid box covering the active ligand binding sites present in the receptor molecule, and all of the residues associated with the binding of the ligand.

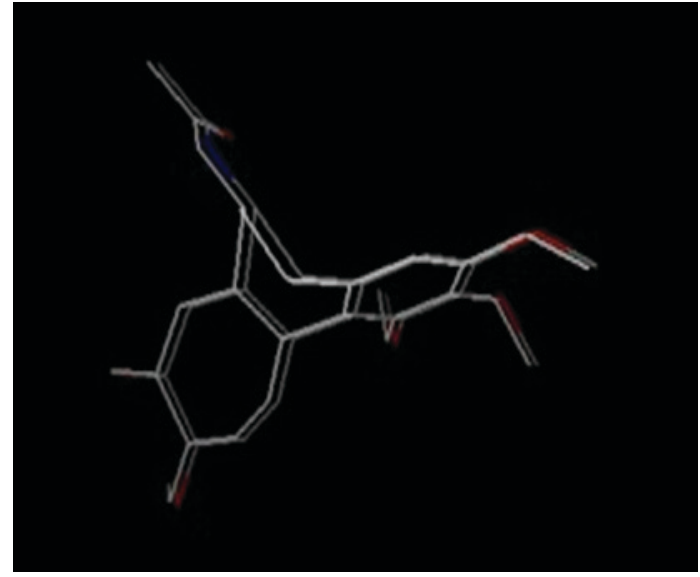

Figure 4. Superimposition of the docked conformation of the colchicine ligand with reference to its bioactive conformation, obtained from the crystal structure of the downloaded protein.

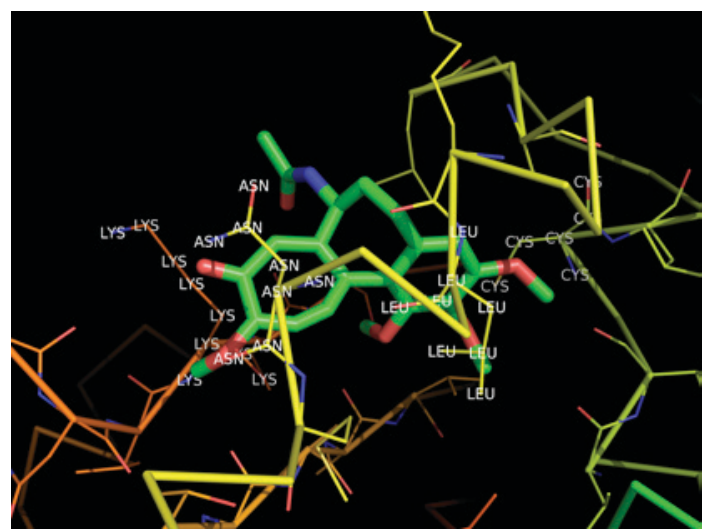

Figure 5. Binding mode and chemical interactions of the bound ligand colchicine within the active ligand binding site of human tubulin protein.

Ligand structures. The ligand was separated from the receptor 3E22.pdb using Chimera software (14). The ligand was docked into receptors using the AutoDock program.

Grid box. The regions of interest used by AutoDock were defined by making a grid box around the active sites. Grid boxes serve an important role in the process of docking as they are designed to cover all of the amino acids present in the active sites which are required for binding, aside from those present in the receptor. The grid box tool uses three thumb wheel widgets, which means that points in the $\mathrm{x}, \mathrm{y}$ and $\mathrm{z}$ dimensions may be altered. The spacing between grid points may be adjusted with an additional thumbwheel. In the present study, the spacing between grid points was fixed at $0.408 \AA$ and the number of points considered was 40,48 and 50 points in the $x, y$, and $z$ dimensions, respectively, and 258.798, 7.947 and 28.673 as $x, y$ and $z$ centers, respectively (8-12).

The results obtained from the molecular docking simulation technique were evaluated by observing the inhibition constant $K i$ of docked molecules using the following method (7):

$\log K i=\Delta \mathrm{G} / \mathrm{RT} * 1 / 2.303$.

$K i=\mathrm{e}$,

$\Delta \mathrm{G} / \mathrm{RT}$, 


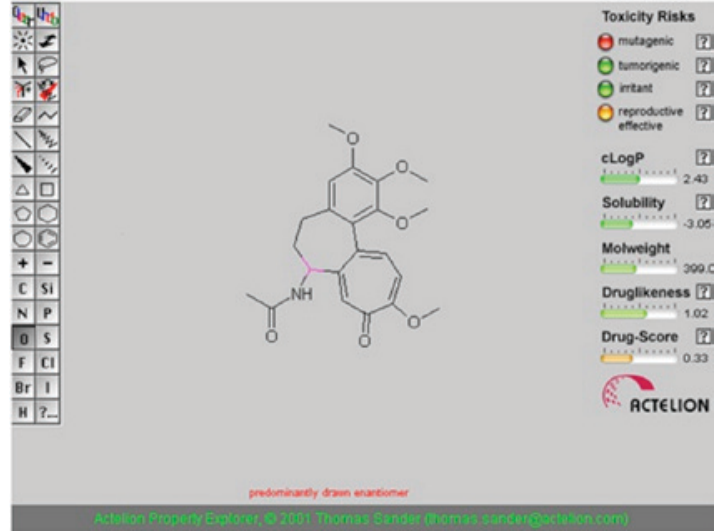

Figure 6. Absorption, distribution, metabolism and excretion-toxicity profiling and toxicity prediction of colchicine.

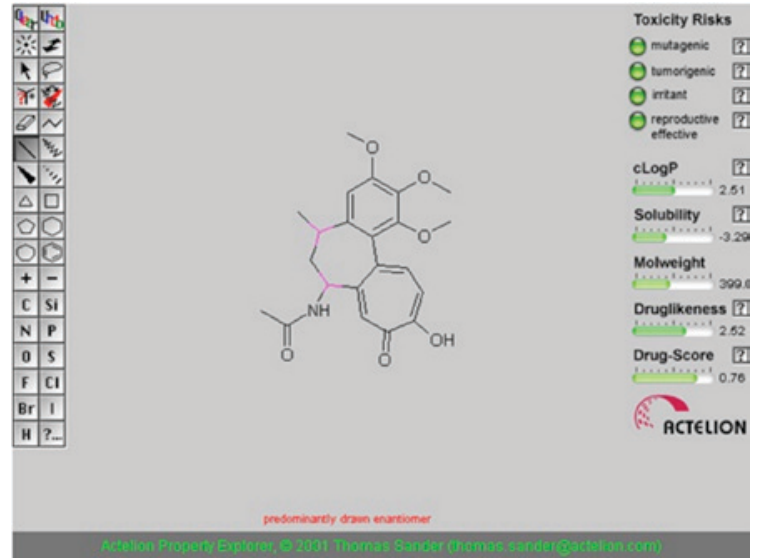

Figure 7. Absorption, distribution, metabolism and excretion-toxicity profiling and toxicity prediction of the modified colchicine derivative Col_1.

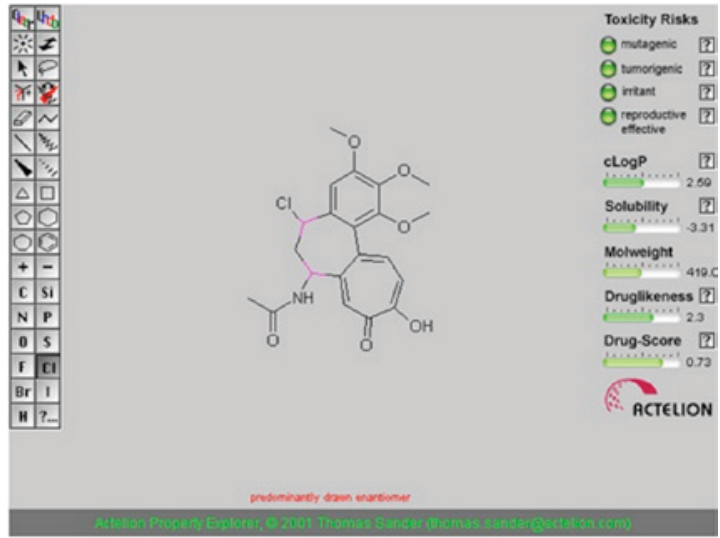

Figure 8. Absorption, distribution, metabolism and excretion-toxicity profiling and toxicity prediction of the modified colchicine derivative Col_2.

$$
K i=\operatorname{antilog} \Delta \mathrm{G} / 1362.97988
$$

Where, $\mathrm{G}=$ binding energy in $\mathrm{kcal} / \mathrm{mol}$; $\mathrm{R}=1.986 \mathrm{kmol}$; $\mathrm{T}=298 \mathrm{~K}$.

Lead modification. The known tubulin protein inhibitor COL was substituted with a number of bioisosteres at different sites

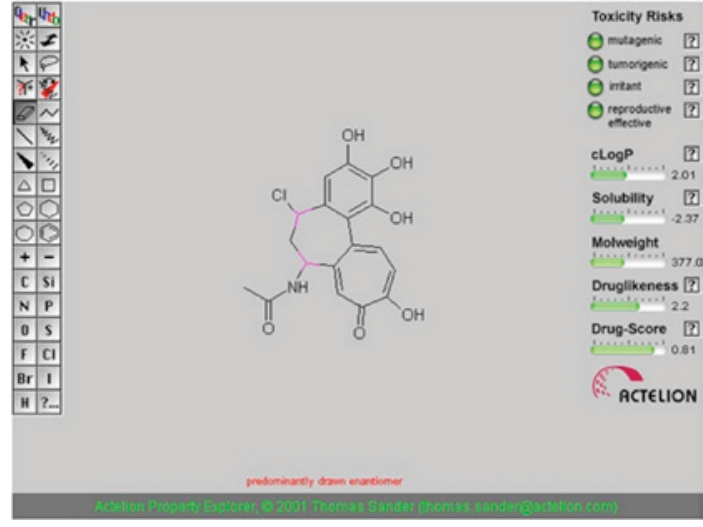

Figure 9. Absorption, distribution, metabolism and excretiontoxicity profiling and toxicity prediction of the modified colchicine derivative Col_3.

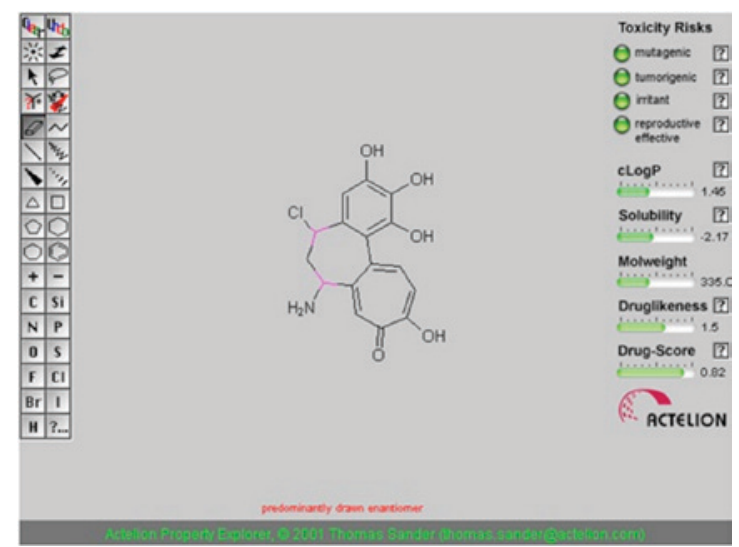

Figure 10. Absorption, distribution, metabolism and excretion-toxicity profiling and toxicity prediction of the modified colchicine derivative Col_4.

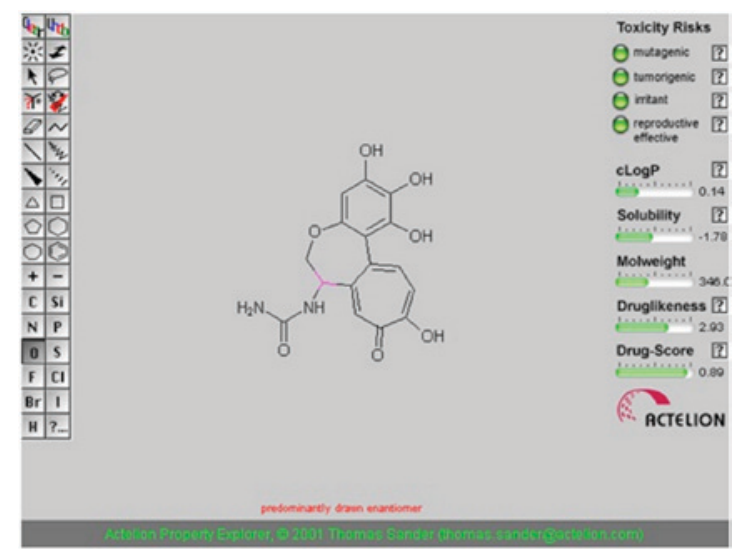

Figure 11. Absorption, distribution, metabolism and excretion-toxicity profiling and toxicity prediction of the modified colchicine derivative Col_5.

to improve the potency and drug-likeness properties, and to reduce toxicity and associated side effects $(15,16)$.

Toxicity and absorption, distribution, metabolism and excretion-toxicity (ADME-T) studies. The modified lead molecules were analyzed using the online program OSIRIS 
(organic-chemistry.org/prog/peo), for the prediction of the presence of any toxic groups or ADME-T properties (17).

\section{Results}

Docking. All of the molecules were docked, and all of the docking studies were validated by checking chemical resemblance, alignment and binding energy (Table I). The grid box covering all the binding residues of the tubulin receptor is presented in Fig. 3.

Validation of the docking process. In order to validate the process of docking a particular ligand with a particular macromolecule, three parameters were considered in the present study.

i) Binding energy. The binding energy of the docked ligand was between -5 and $-15 \mathrm{kcal} / \mathrm{mol}$.

ii) Overlay. The docked conformation of ligand was overlaid with the crystal structure of the downloaded protein. Testing of the Autodock docking algorithm with COL (already present within the receptor as a complex) was completed successfully and the docked confirmation of COL was exactly superimposed with the reference structure of COL, i.e. its crystal structure. The docked conformation of COL overlaid upon the bioactive conformation is presented in Fig. 4. The re-docking of COL was successfully achieved in order to refine the results.

iii) Interactions. Consistent interactions between the docked ligand and the receptor must be observed following docking, compared with the interactions present in the crystallized structure of the protein. The interactions observed in the present study between the bound ligand COL and the binding residues present in the tubulin receptor are presented in Fig. 5. All of the bonds of the ligands remained flexible, while in receptor 3E22, ALA15 (alanine-15) and ASN228 (asparagine-228) were made flexible. All of the bonds of ALA15 and ASN228 remained rotatable (8-12).

Toxicity and ADME-T studies. The results obtained from the toxicity and ADME-T analysis in the present study are exhibited in Figs. 6-11. The results demonstrated that all 5 of the colchicines-based designed tubulin inhibitors were free from any major toxic effects, including mutagenicity, tumorogenicity, irritant as well as reproductive effects, as the online program OSIRIS (Molecular Property Explorer), did not identify the presence of any toxic functional groups responsible for these toxic effects, within the designed molecules. All of the molecules had excellent pharmacokinetic profilesand were strictly filtered using Lipinski's Rule of Five (18) for their physicochemical properties.

\section{Discussion}

The results of the present study demonstrated that the novel colchicine-derived inhibitors of human tubulin protein may be potent drugs for the treatment of arthritic disease. All of the designed inhibitors exhibited good binding affinity with the macromolecule as demonstrated by the values of the inhibition constant $\mathrm{Ki}$.

The toxicity and ADME-T analyses of all of the designed inhibitors confirm the absence of any toxic functional groups. All of the inhibitors were designed in accordance with Lippinski's Rule of Five (18); therefore, all of them exhibited good drug and drug-likeness scores.

All of the designed COL-derived lead molecules exhibited increased binding affinity compared with COL, in addition to improved physico-chemical properties compared with the parent molecule COL.

\section{References}

1. Bettschen J: Gouty arthritis: Current treatments and new developments. BSP Candidate: e1-e8, 2010.

2. Talbott JH: Diagnosis and treatment of gouty arthritis. Calif Med 79: 220-226, 1953.

3. Jordan KM, Cameroon JS, Snaith M, Zhang W, Doherty M, Seckl J, Hingorani A, Jaques R and Nuki G; British Society for Rheumatology and British Health Professionals in Rheumatology Standards, Guidelines and Audit Working Group (SGAWG): British society for rheumatology and British health professionals in rheumatology guideline for the management of gout. Rheumatology (Oxford) 46: 1372-1374, 2007.

4. Peter-Ruiz F: Treating to target: A strategy to cure gout. Rheumatology (Oxford) 48 (Suppl 2): ii9-ii14, 2009.

5. Forli Stefano: Raccoon Autodock VS preparation tool. Scripps Res Institute, 2009.

6. Tuccinardi T, Cascio MG, Marzo V Di, Manera C, Ortore G, Saccomanni G and Martinelli A: Structure-based virtual screening: Identification of novel $\mathrm{CB}_{2}$ receptor ligands. Bioorgan Med Chemistry Lett: 15-19, 2007.

7. Mujwar S and Pardasani KR: Prediction of Riboswitch as a potential drug target for infectious diseases: An Insilico case study of anthrax. J Med Imag Health Informat 5, 2015.

8. Sundararajan S, Balajee R, Rajan M and Dhana S: Comparative docking analysis of neuraminidase with various inhibitors. Int J Pharmacy Pharmaceutical Sci 2: 83, 2010.

9. Morris GM, Huey R, Lindstrom W, Sanner MF, Belew RK, Goodsell DS and Olson AJ: Autodock4 and AutoDockTools4: Automated docking with selective receptor flexibility. J Comput Chem 30: 2785-2791, 2009.

10. Phoristhong N: Molecular docking study on anticancer activity of plant-derived natural products. Med Chemistry Res 19: 817-835, 2010.

11. Gueto C, Torres J and Vivas-Reyes R: CoMFA, LeapFrog and Blind docking studies on sulfonanilide derivatives acting as selective aromatase expression regulators. Eur J Med Chem 44: 3445-3451, 2009.

12. Mujwar S and Pardasani KR: Prediction of riboswitch as a potential drug target in Streptococcus. Online J Bioinformatics 12, 2012.

13. Berman HM, Westbrook J, Feng Z, Gilliland G, Bhat TN, Weissig H, Shindyalov IN and Bourne PE: The protein data bank. Nucleic Acids Res 28: 235-242, 2000.

14. Pettersen EF, Goddard TD, Huang CC, Couch GS, Greenblatt DM, Meng EC and Ferrin TE: UCSF Chimera-a visualization system for exploratory research and analysis. J Computat Chem 25: 1605-1612, 2004.

15. Patani AG and LaVoie EJ: Bioisosterism: A rational approach in drug design. Chem Rev 96: 3147-3176, 1996.

16. Hansch C, Garg R and Kurup A: Searching for allosteric effects via QSAR. Bioorg Med Chem 9: 283-289, 2001.

17. Sander T, Freyss J, von Korff M, Reich JR and Rufener C: OSIRIS, an entirely in-house developed drug discovery informatics system. J Chem Inf Model 49: 232-246, 2009.

18. Lipinski CA, Lombardo F, Dominy BW and Feeney PJ: Experimental and computational approaches to estimate solubility and permeability in drug discovery and development settings. Adv Drug Deliv Rev 46: 3-26, 2001 